\title{
Manipulability Analysis of Kicking Motion in Soccer Based on Human Physical Properties
}

\author{
Yoshiyuki Tanaka, Mitsuhisa Shiokawa, Hiroyuki Yamashita, and Toshio Tsuji
}

\begin{abstract}
This paper presents an analysis tool of kicking motion in soccer based on robotic techniques. The developed tool can calculate kinematics, dynamics, and manipulability of trainee's movements with considerations of human physical characteristics from the trainee's postures measured by a motion capture system, and can visually provide the quantitative analysis to users in the main window of the tool. The effectiveness of the proposed approach is verified through basic experiments with skilled and unskilled subjects in soccer.
\end{abstract}

\section{INTRODUCTION}

A human performs a variety of skillful and rapid movements in sports by actively adjusting dynamic characteristics of his/her musculoskeletal system with a large number of the degree of freedom according to a target task. That is, such player's skills in sports are determined by the regulation ability of his/her dynamic characteristics desired for the task. In the field of sports, a coaching is generally provided on the basis of the experience of coaches and the forms of major players measured in sports. However, it is difficult for trainees to thoroughly master the desired forms and techniques due to the differences in the comprehension of trainee and the explanation of coaches. Quantitative analysis of desired movements, as well as trainee's movements, in sports would be useful for designing quantitative instructions by the coaches.

Many studies have been conducted on the analysis of the kicking motion in soccer. For example, Nunome et al.[1] measured the 3D coordinates of the kicking leg during soccer shooting, and examined the difference on the leg movements between curve and straight ball soccer shooting. William et al. [2] examined the differences in soccer kicking depending on the gender of soccer players. Dorge et al. [3] examined the differences between the preferred and non-preferred leg. However, these previous studies report kinematic features focusing mainly on the postures. Nunome et al. [4] measured the $3 \mathrm{D}$ coordinates of the kicking leg in the case of inside kicking and estimated the joint torque generated in the lower limbs. Asai et al.[5] estimated the fundamental characteristics that cause ball rotation by using models of the kicking leg and the ball surface. These kinetic analyses focus on the force

This work was supported in part by a Grant-in-Aid for Scientific Research from the Japanese Ministry of Education, Science and Culture (16760203 and 15360226).

Y. Tanaka, H. Yamashita, and T. Tsuji are with Department of Artificial Complex Systems Engineering, Graduate School of Engineering, Hiroshima University, 1-4-1 Kagamiyama, Higashi-hiroshima, JAPAN ytanaka@bsys.hiroshima-u.ac.jp

M. Shiokawa is with Faculty of Health and Welfare, Prefectural University of Hiroshima, 1-1 Gakuencho, Mihara, JAPAN siokawa@puhiroshima.ac.jp and joint torque of the particular parts of a human body although soccer players control the whole body to realize desired movements for kicking the ball. As results, it may be difficult for users to understand the analyzed results of dynamic movements by these previous researches.

On the other hand, it has been considered that a human would design his/her limb's motion in the operational task space [6]. Accordingly, it will be useful for developing an efficient training method if the skills required for target motion can be expressed quantitatively and visually in the operational task space so that the user can instinctively grasp the sense of analyzed results.

In the field of robotics, there are several methods to evaluate the manipulability of robots from the kinematical and dynamical viewpoints [7][8]. These methods can provide a quantitative analysis of the robot based on its link configurations and have been applied to the analysis of human multi-joint movements [9], the manipulability evaluation of welfare equipments [10]. The previous methods, however, cannot consider any biological characteristics of human motor movements. On the other hand, we developed a quantitative evaluation method using human force manipulability inspired by human joint-torque characteristics and applied to the layout problem of driving interfaces with consideration of human motor ability [11][12]. The aim of this study is to develop a novel analysis approach using human force manipulability for sports training.

This paper proposes the analysis system of kicking motion in soccer by using robotic techniques with consideration of human physical properties. The developed system can calculate kinematics, dynamics, and manipulability just from the trainee's postures measured by a motion capture system, and can visually provide the quantitative analysis to the user. This paper is organized as follows: Section II describes an outline of the developed tool for analyzing kicking motion in soccer. Section III explains human force manipulability and human joint torque characteristics of the lower extremities measured. Finally, Section IV shows the effectiveness of the proposed method for soccer analysis from experiments with skilled and unskilled subjects.

\section{Analyzer Tool of Kicking Motion in Soccer}

Fig. 1 represents the general procedure of analyzing the soccer kicking motion using the developed tool.

The tool starts to compute joint angles in a human model of the whole body expressed by the combination of multijoint links from the measured data by the motion capture system. Fig. 2(a) shows the structure of a human model 


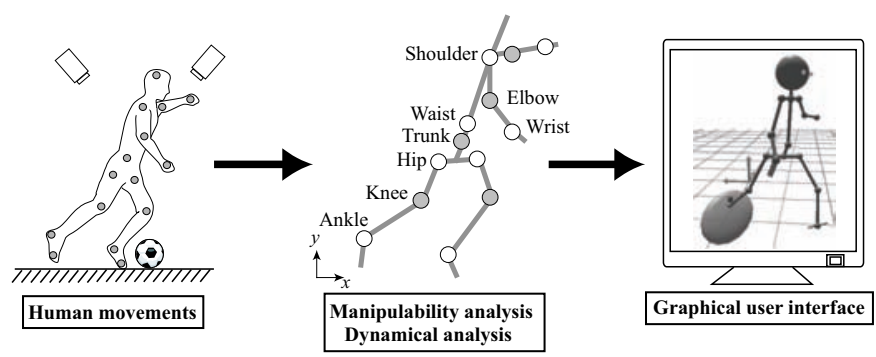

Fig. 1. Analysis of the kicking motions in soccer

utilized in this paper, in which the total number of degree-offreedom (DOF) is 34. Each upper extremity has three links with seven DOF, each lower extremity has also three links with seven DOF, and the trunk has three links with six DOF. The basic posture of the human model is defined as shown in the figure.

The quantitative analysis of human motion is performed by using a set of the computed joint angles with consideration of human physical properties. Fig. 2(b) expresses the main part of the analysis tool. The database of human physical properties stores the information on inertia and mass of body parts depending on the tall and weight of a subject presented in [13], and the model of the experimentally measured jointtorque characteristics of the human extremities. Dynamics of human movements are analyzed in the dynamic simulator (Adams, MSC Software Corporation), while kinematics and manipulability are analyzed in the programmed simulator (Matlab, The MathWorks, Inc.).

The analysis results are then presented with the animation of measured human motion on the feedback viewer so that users can instinctively understand time-variant properties of kicking motion. Fig. 2(c) shows the graphical user interface programmed by Microsoft Visual $\mathrm{C}++$ and Visual Basic. The changes of postures and ellipsoids can be seen from any point by operating the control buttons for changing the point of view.

\section{HUMAN FORCE MANIPULABILITY FOR THE LOWER EXTREMITIES IN KICKING MOTION}

\section{A. Human force manipulability [11]}

A human can control the end-point force $f \in \Re^{m}$ by regulating the joint torque $\tau \in \Re^{n}$ and the posture $\boldsymbol{\theta} \in \Re^{n}$. The relationship between the end-point space and the joint space is given by

$$
\boldsymbol{\tau}=\boldsymbol{J}^{T}(\boldsymbol{\theta}) \boldsymbol{f}
$$

where $\boldsymbol{J}(\boldsymbol{\theta}) \in \Re^{m \times n}$ is the Jacobian matrix on the end-point position $\boldsymbol{x} \in \Re^{m}$ with respect to $\boldsymbol{\theta}$.

Let us introduce the joint torque activation level $\boldsymbol{\alpha}=$ $\left(\alpha_{1}, \alpha_{2}, \cdots, \alpha_{n}\right)^{\mathrm{T}} \in \Re^{n}$, in which $\left|\alpha_{i}\right| \leq 1$ represents the ratio of $i$-th joint torque to the maximum torque under the maximum voluntary contraction (MVC) and its sign denotes the joint rotational direction (the flexional direction is defined as positive). The joint torque vector $\tau$ is then expressed with $\boldsymbol{\alpha}$ because the muscle tension is proportional to its muscle

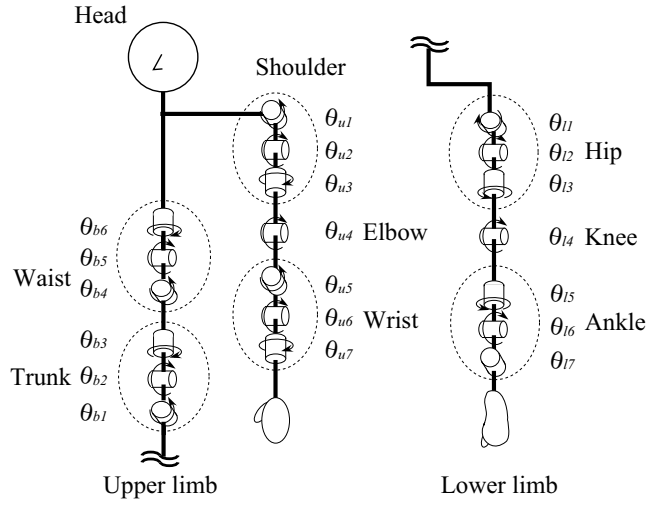

(a)

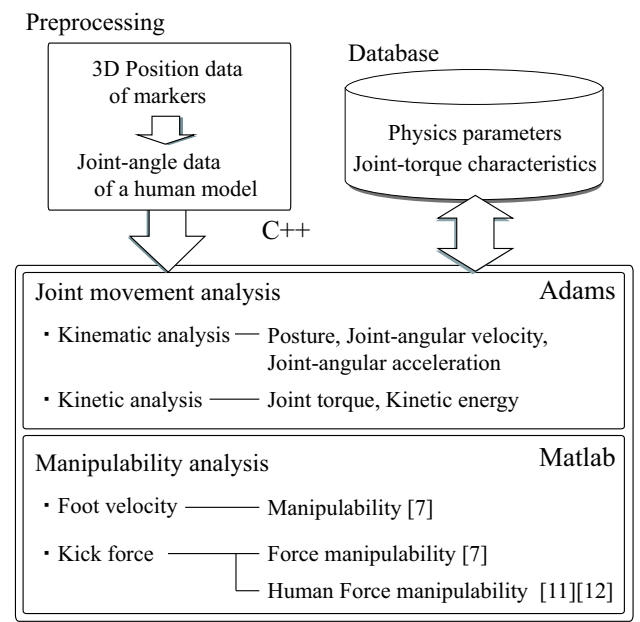

(b)

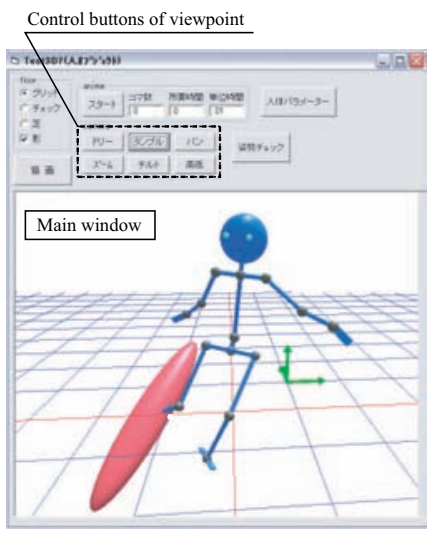

(c)

Fig. 2. Details of the developed analysis tool for kicking motion

activation level [6] as follows:

$$
\tau=T(\theta) \alpha
$$

where $\boldsymbol{T}(\boldsymbol{\theta})=\operatorname{diag} .\left(\tau_{1 j}^{\max }\left(\theta_{1}\right), \tau_{2 j}^{\max }\left(\theta_{2}\right), \cdots, \tau_{n j}^{\max }\left(\theta_{n}\right)\right)$ $\in \Re^{n \times n}(j \in\{n, p\}$; the suffix $n$ and $p$ indicate the negative and the positive direction, respectively). Each diagonal element of $\boldsymbol{T}(\boldsymbol{\theta})$ indicates an absolute value of the maximum joint torque at the angle $\boldsymbol{\theta}$. Substituting (1) into (2), $\boldsymbol{\alpha}$ can 
be represented with $f$ as follows:

$$
\boldsymbol{\alpha}=\boldsymbol{T}^{-1} \boldsymbol{J}^{T} \boldsymbol{f} .
$$

A set of the force $f$ generated by the muscles within $\|\boldsymbol{\alpha}\| \leq 1$ makes an $m$ dimensional ellipsoid represented by

$$
\boldsymbol{f}^{\mathrm{T}}\left(\boldsymbol{J} \boldsymbol{T}^{-1}\right)\left(\boldsymbol{J} \boldsymbol{T}^{-1}\right)^{\mathrm{T}} \boldsymbol{f} \leq 1 .
$$

The ellipsoid given in (4) is corresponding to the modified Force Manipulability Ellipsoid (FME) [7] with the matrix $\boldsymbol{T}$ which reflects the human joint-torque characteristics. Similar to the case of the FME, the shape of this human FME represents a performance index in generating force according to the operational direction.

\section{B. Joint-torque Characteristics of the Lower Extremities}

Measurement experiments of joint-torque characteristics of the right leg were carried out along the link model defined in Fig. 2 with four male subjects (University students, aged 22-24).

Fig. 3 shows the experimental apparatus for investigating human joint characteristics during uniarticular movements, which consists of the measurement part of joint torque and EMG signals, and the bio-feedback display for monitoring the muscle activation level during experiments. BIODEXSYSTEM-2AP (MEDICAL SYSTEMS INC.; the maximum torque is $610[\mathrm{Nm}]$ ) was employed for measuring joint torque properties in the experiment (See Fig. 3). Assembling some attachments according to the target joint movements, joint torque or end-point force can be measured. The EMG signals were measured with an amplifier (NEC Medical Systems, MT11) and a set of disposable electrodes (GE Mamrquette Medical Systems Japan, Biorode SDC-H). The measured EMG signals were rectified and integrated with the data during the past 0.1 seconds, and normalized with the values measured in the MVC at the middle angle of the range of joint motion. The normalized values were utilized as the muscle contraction levels in this study.

In the experiments, a subject was instructed to generate his/her joint torque under the $40 \%$ muscle contraction level for 10 seconds by monitoring the biofeedback display in front of him. Joint torque in each uniarticular movements is measured twice at each joint angle changed by 0.349 [rad] around the middle of joint excursion. The measured data were processed along the following procedure as:

1) Divide the measured signals during 10 seconds by every second for each trial, and calculate the average and standard deviation of the muscle contraction level in every second,

2) Extract 10 periods in which the muscle contraction level is close to $40 \%$ from the 20 periods created in the previous process,

3) Calibrate the averaged joint torque of the extracted sections to one at the $40 \%$ muscle contraction level,

4) Calculate the average and standard deviation of the calibrated joint torques.

Fig. 4 shows examples of the joint-torque properties measured by Subject A, where the solid line with white circles

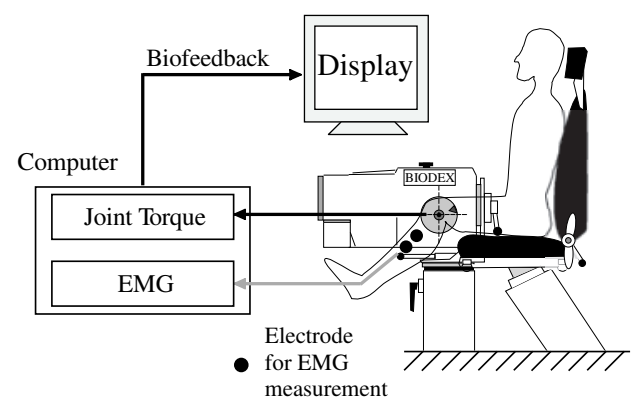

(a) Measuring system

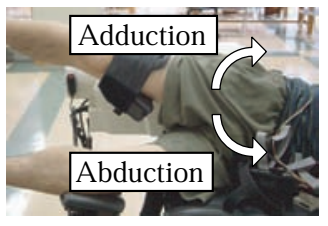

Hip Abduction/Adduction

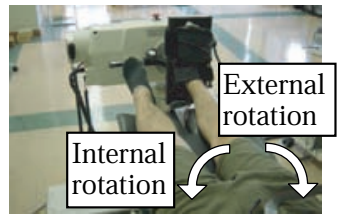

Hip Internal/External rotation

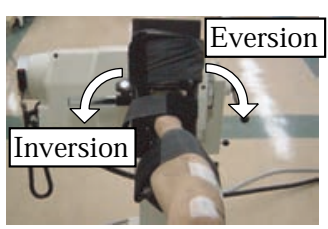

Ankle Inversion/Eversion

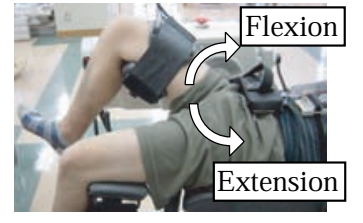

Hip Flexion/Extension

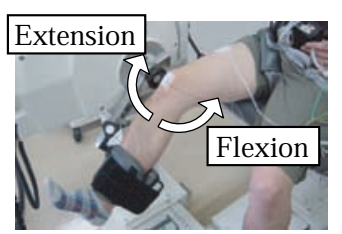

Knee Flexion/Extension

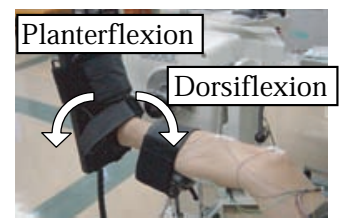

Ankle Planterflexion/Dorsiflexion (b) Attachment for measuring joint-torque

Fig. 3. Experimental apparatus for measuring human joint torque

represents the joint torque in the abduction in the figure (a), flexion in (b) and (d), internal rotation in (c), inversion in (e), planterflexion in (f), while a dotted line with black circles in the adduction in the figure (a), extension in (b) and (d), external rotation in (c), eversion in (e), dorsiflexion in (f). As shown in the experimental results, the characteristics of human joint-torque is influenced by the joint angle and the rotational direction. Similar tendency was shown about the other three subjects.

To introduce the measured human joint-torque properties into human force manipulability, the maximum joint-torque in uniarticular movements under the MVC, $\tau_{i j}^{\max }\left(\theta_{i}\right)$, was calculated from the measured data at $40 \%$ muscle contraction level by magnifying two and a half times under the assumption that joint-torque is almost linear to the muscle activation level of the corresponding agonist. In this paper, $\tau_{i j}^{\max }\left(\theta_{i}\right)$ was modeled with the second-order polynomial equation with respect to the joint angle as:

$$
\tau_{i}^{\max }\left(\theta_{i}\right)=a_{2} \theta^{2}+a_{1} \theta+a_{0}
$$




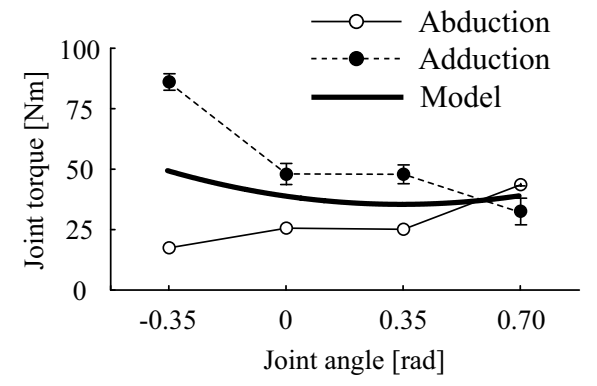

(a) Hip abduction and adduction

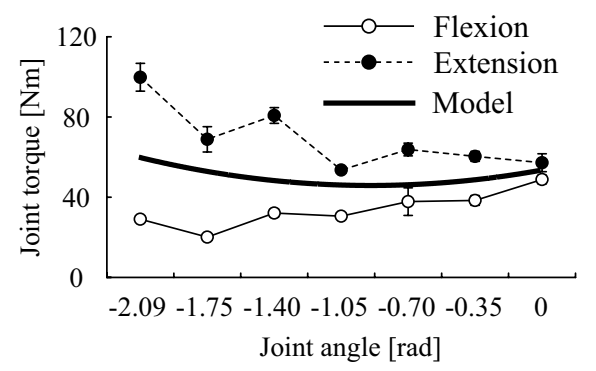

(d) Knee flexion and extension

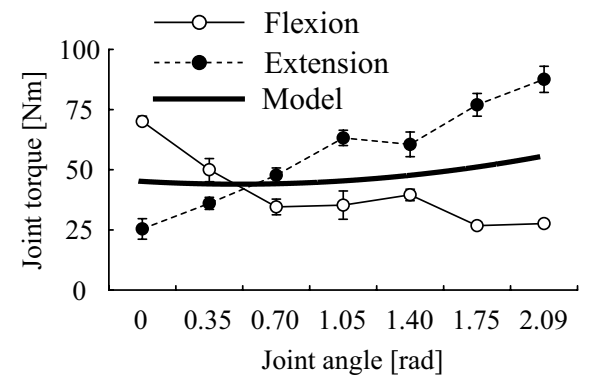

(b) Hip flexion and extension

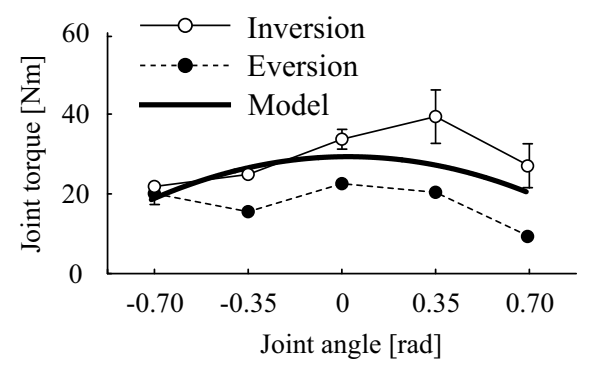

(e) Ankle inversion and eversion

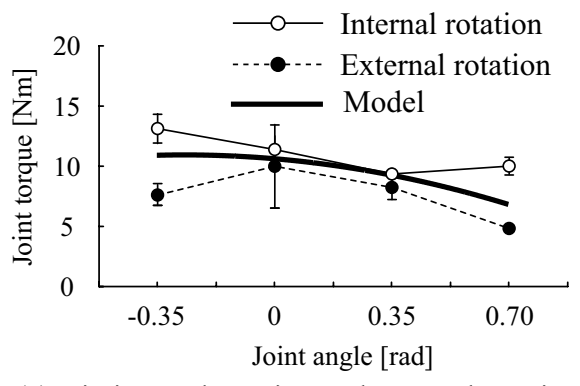

(c) Hip internal rotation and external rotation

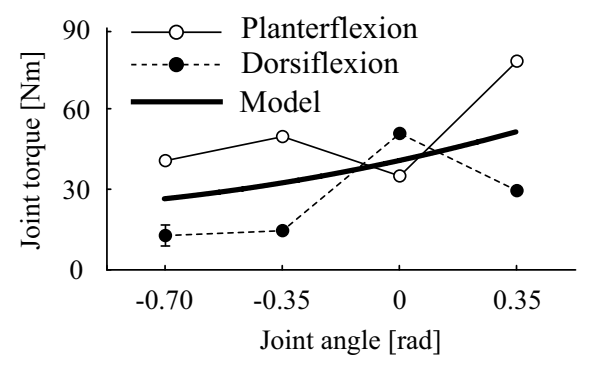

(f) Ankle planterflexion and dorsiflexion

Fig. 4. Joint-torque characteristics with respects to joint-angle in the lower limbs (Subject A)

where the coefficients $a_{0}, a_{1}$, and $a_{2}$ were estimated by fitting the magnified data with the least squares method. The thick line in Fig. 4 represents the model of joint-torque properties on each of uniarticular movements.

\section{Analysis of the Kicking Motion in Soccer}

Analysis of kicking motion was conducted along the proposed approach with two skilled subjects in soccer (Subject A: aged 21, height $170[\mathrm{~cm}]$, weight $68.0[\mathrm{~kg}]$; Subject B: aged 22, height $173[\mathrm{~cm}]$, weight $65.0[\mathrm{~kg}])$ and two unskilled subjects (Subject C: aged 23, height $175[\mathrm{~cm}]$, weight 72.0 [kg]; Subject D: aged 22, height 179 [cm], weight $60.0[\mathrm{~kg}])$.

\section{A. Experimental method}

Fig. 5 shows the experimental apparatus for measuring the kicking motion, in which VICON (OXFORD METRICS; sampling frequency: $120[\mathrm{~Hz}]$ ) was employed to capture rapid movements of subjects. The subjects were asked to kick a ball forward by their instep within two strides from the initial position because of the measuring range of the motion capture, in which the approach angle $\varphi$ was fixed at about 30 [deg.] (See Fig. 5(b)). In the experiment, 28 markers were attached on the whole body of the subject so that all joint angles defined in Fig. 2 can be calculated from the 3D positional information of the markers.

\section{B. Experimental results}

Fig. 6 shows a series of postures during instep kicking motion in stick picture for the skilled player (Subjects B) and the unskilled player (Subject D ), where the time axis at impact is set as 0 [s]. It can be found that the skilled player takes less time to kick the ball as compared to an unskilled

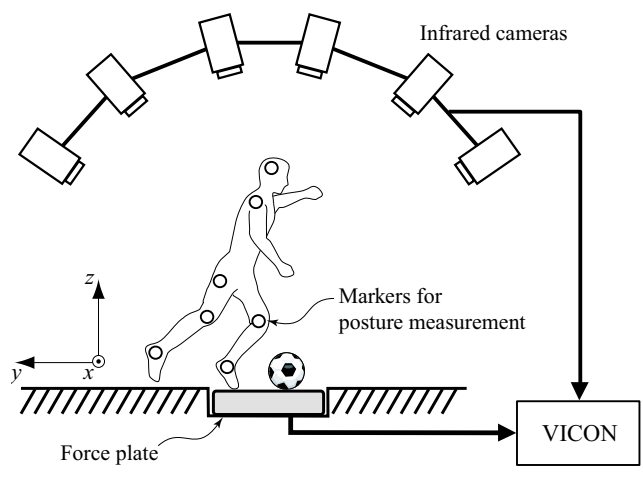

(a) Side view

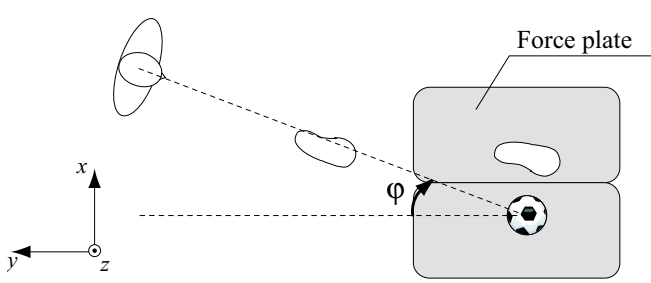

(b) Top view

Fig. 5. Experimental apparatus for measuring kicking motion in soccer

one. Further analysis of kicking motion was carried out with the measured postures by the developed tool.

Fig. 7 shows the time histories of kinematical and dynamical properties during kicking motion for the skilled player (Subject B) and the unskilled player (Subject D). The figure (a) is the results on the toe velocity, the figures (b) and (c) on the rotational energy of the upper and lower torso where the first peak is represented with an inverted triangle, the figures 


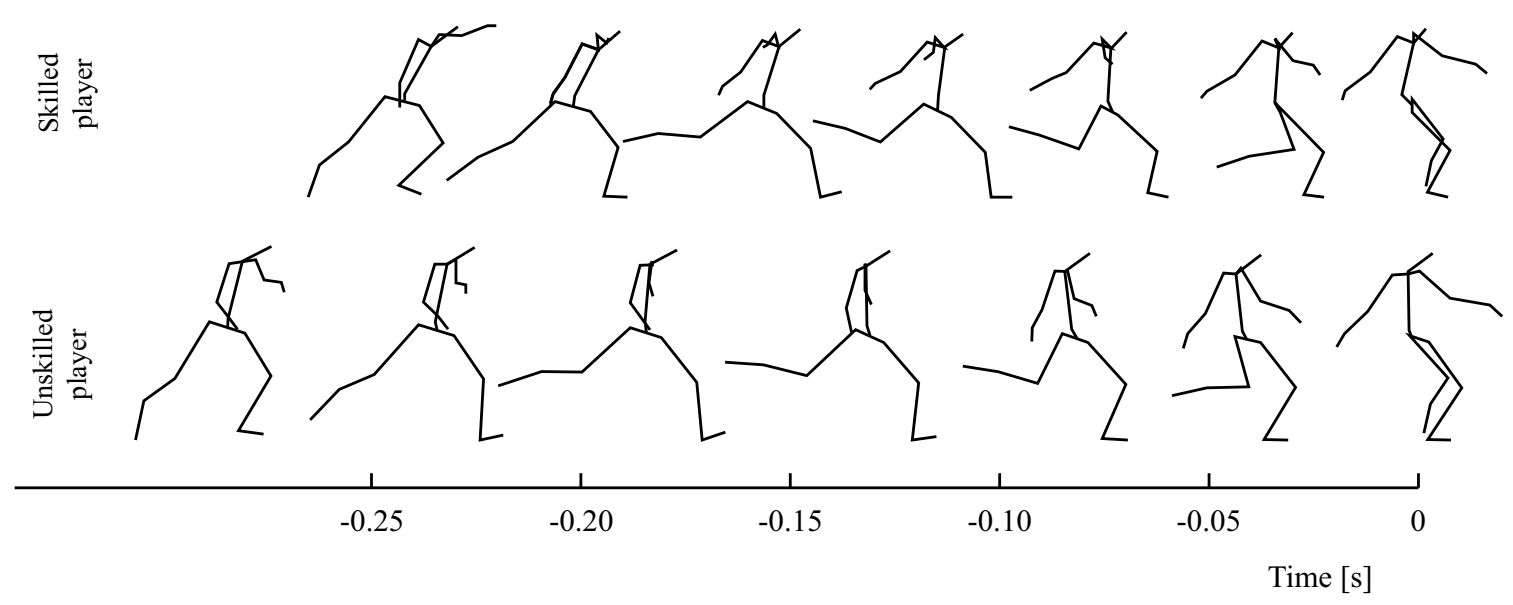

Fig. 6. A series of postures during instep kicking motion for the skilled and unskilled subjects

(d) and (e) on the manipulability and force manipulability of the kicking leg toward the ball.

There exist small differences between the toe velocities between the skilled and unskilled players as shown in Fig. 7(a). They increase the velocity for kicking the ball, and the mean of velocities at impact is almost same (skilled player: $17.681 \pm 0.567[\mathrm{~m} / \mathrm{s}]$, unskilled player: $17.484 \pm$ $0.731[\mathrm{~m} / \mathrm{s}])$. On the other hand, distinctive differences can be observed on the rotational energy of the upper and lower torso during kicking motion as shown in Fig. 7(b) and (c). The maximum value of rotational energy by the skilled player is larger than that by the unskilled player, and a period between the peaks of rotational energy of the upper and lower torso, $\Delta t$, tends to be shorter. Skilled players could efficiently and rapidly transmit large rotational energy generated by the upper torso, as well as the dual-arm, to the lower torso for kicking motion.

The above kinematical and dynamical results demonstrate that the player controls the whole body to realize desirable kicking motion. Finally, in association of these results, the meaning lying behind kicking motion is discussed in view of the manipulability of the kicking leg by the skilled subject presented in Fig. 7(d) and (e).

The computed manipulability for the skilled player increases and has a peak at the impact $t=0[\mathrm{~s}]$, while the computed force manipulability increases around the transmitting period of rotational energy. He bends his knee to generate larger kick force with synchronizing the transmission of rotational energy so that the end-point of the leg can accelerate toward a ball, and stretches his leg to maximize the toe velocity at the impact with the ball. These two points should be focused in evaluating the leg movements during instep kicking.

\section{Evaluation results}

To provide the quantitative evaluation to trainee, we selected five indexes based on the experimental results: i) Velocity of the toe point at impact $E_{v}$; ii) Manipulability at impact $E_{m}$; iii) Force manipulability at its peak $E_{f}$; iv) Rotational energy of the lower torso at its peak $E_{r}$; v) Inverse of a period $\Delta t$. It would be more appropriate to increase the value of all indexes.

Figs. 8 show the evaluation results of the skilled players (Subjects A, B) and the unskilled players (Subjects C, D) on a radar chart, respectively. The charts of the skilled players are larger, while those of the unskilled players are smaller with an awkward shape. It is contemplated that the balance of the entire body movement can be determined by inspecting the shape of the radar chart designed in this paper.

Accordingly, it is possible to analyze the kicking motion of the entire body movement and represent the characteristics of kicking motion in soccer by using the proposed approach.

\section{Conclusion}

The present paper developed the analysis tool of kicking motion in soccer just by using captured postures that can provide the effective and efficient training information with trainees as well as coaches. The experiments were conducted to investigate the kicking motion in soccer with regard to the skilled and unskilled players and showed the following major points as:

- Both the lower and upper torsos are equally important in soccer kicking.

- Manipulability increases to maximize the end-point velocity at impact.

- Force manipulability of the lower extremities increases around the transmitting period of rotational energies of the body

Future research will be directed toward introducing the inertia ellipsoid and examining the relationship between the kicking motion of trainees and the dynamic behaviors of kicked ball. Further, we plan to establish an evaluation method with more subjects and finally examine its application to coaching in the field.

\section{REFERENCES}

[1] H. Nunome, K. Matsunaga, H. Yamamoto, "A Three-Dimensional Analysis of the Leg Motion during a Crve Ball Soccer Shooting," Japanese Journal of Sports Sciences, Vol.16, No.1, pp.105-110, 1997 (In Japanese). 


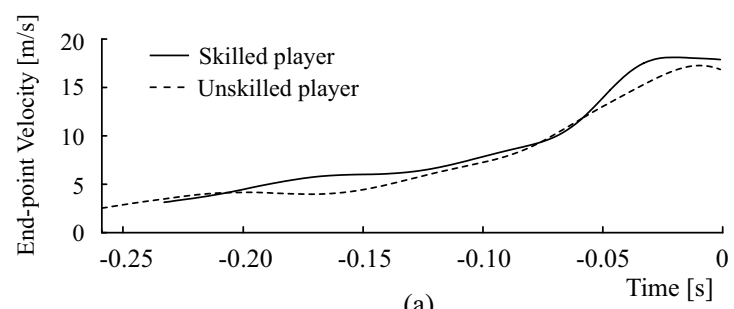

(a)

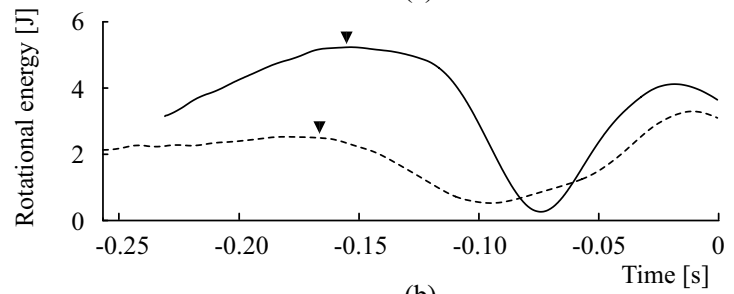

(b)

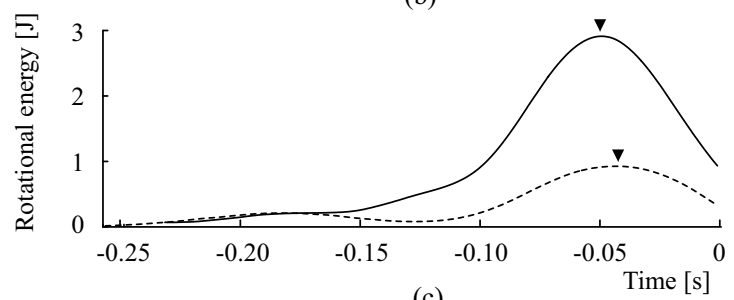

(c)

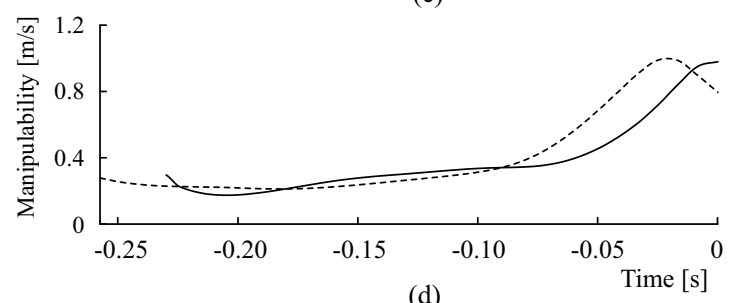

(d)

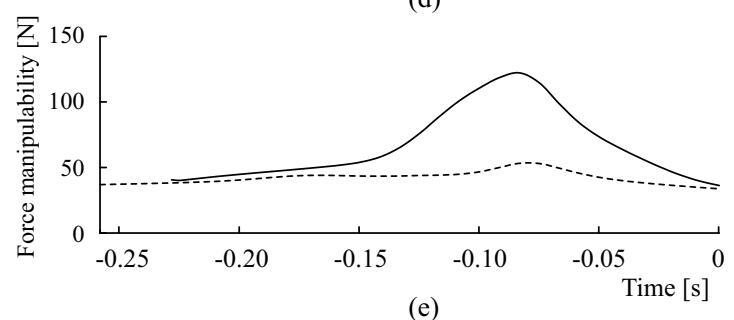

(e)

Fig. 7. Examples of the time histories of kicking motion for the skilled and unskilled subjects

[2] William Roy Barfield, Donald T.Kirkendall, and Bing Yu, "Kinematic Instep Kicking Differences between Elite Female and Male Soccer Players," Journal of Sports Science and Medicine, Vol. 1, No. 1, pp. 72-79, 2002.

[3] H.C.Dorge, T. Bull Andersen, and E.B.Simonsen, "Biomechanical Differences in Soccer Kicking with the Preferred and the Nonpreferred Leg," Journal of Sports Sciences, Vol. 1, No. 20, pp. 293-299, 2002.

[4] H. Nunome, "Biomechanics of Kicking motion in soccer," Japanese Journal of Biomechanics in Sports and Exercise, Vol.3, No.2, pp.104110, 1999 (In Japanese).

[5] T. Asai, "Comparison between Instep Kick and Curve Kick in Soccer," Japanese Journal of Biomechanics in Sports and Exercise, Vol.3, No.2, pp.111-118, 1999 (In Japanese).

[6] K. Ito, M. Ito, "Control of human and robot movement," The Society of Instrument and Control Engineers, 1991.

[7] T. Yoshikawa, "Measue of Manipulatability for Robot Manipulations," Journal of the Robotics Society of Japan, Vol.2, No.1, pp.63-67, 1984.

[8] H. Asada, "Geometrical Representation of Manipulator Dynamics and Its Application to Arm Design," Transaction of the ASME, Journal of Dynamic Systems, Measurement, and Control, Vol.105, pp.105-135,

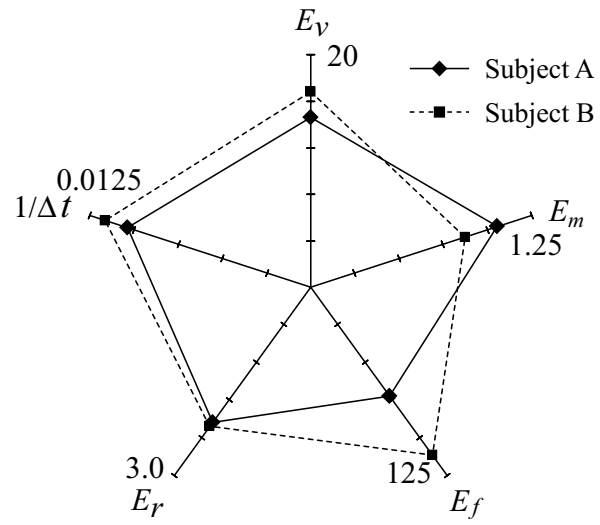

(a) Skilled players

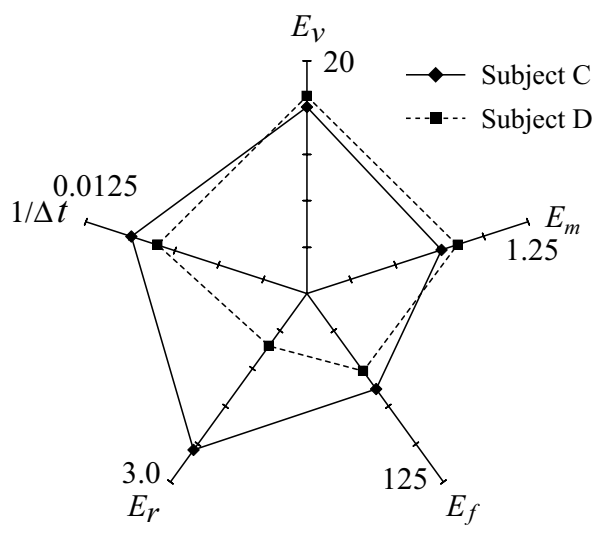

(b) Unskilled players

Fig. 8. Evaluation results of instep kicking motion for the four subjects

1983.

[9] K. Ota et al. "Analysis of Human Movement under Environmental Constraints -Adaptability to Environment during Crank Rotation Tasks," The Institute of Electronics, Information and Communication Engineers D-II, Vol. J81-D-II, No.6, pp.1392-1401, 1998 (In Japanese).

[10] A. Otsuka, T. Tsuji, O. Fukuda, M. E. Shimizu, M. Sakawa, "Development of an internally powered functional prosthetic hand with a voluntary closing system and thumb flexion and radial abduction," Proc. of the 2000 IEEE International Workshop on Robot and Human Interactive Communication, pp.405-410, 2000.

[11] Y. Tanaka, N. Yamada, I. Masamori, and T. Tsuji, "Manipulability Analysis of lower Extremities Based on Human Joint-Torque Characteristics," Transaction of the Society of Instrument and Control Engineers, Vol.40, No.6, pp.612-618, 2004 (In Japanese).

[12] Y. Tanaka, N. Yamada, K. Nishikawa, I. Masamori, and T. Tsuji, "Manipulability Analysis of Human Arm Movements during the Operation of a Variable-Impedance Controlled Robot," Proceedings of the 2005 IEEE/RSJ International Conference on Intelligent Robotics and Systems, pp. 3543-3548, 2005.

[13] M. Ae, "The Inertia Coefficient of Body Segments of Japanese Boys and Athletes,' Japanese Journal of Science, Vol.15, No.3, pp.155-162, 1996 (In Japanese). 